\title{
Phase II Trial of Acai Juice Product in Biochemically Recurrent Prostate Cancer
}

Integrative Cancer Therapies 2018, Vol. 17(4) II03-1 108 (C) The Author(s) 2018 Article reuse guidelines: sagepub.com/journals-permissions DOI: 10.1 I77//5347354/8803755 journals.sagepub.com/home/ict

@SAGE

\author{
Elizabeth R. Kessler, MD', Lih-Jen Su', Dexiang Gao, PhD', \\ Kathleen C. Torkko, PhD', Michael Wacker', Mary Anduha', \\ Nicole Chronister', Paul Maroni, MD', E. David Crawford, MD', \\ Thomas W. Flaig, MD', L. Michael Glode, MD', and Elaine T. Lam, MD'
}

\begin{abstract}
Background: Plant derivatives have been studied as therapies for prostate cancer based on their purported antiinflammatory and antioxidant properties and low toxicities. The acai berry is an example of a plant rich in phytochemicals, which may slow the growth of prostate cancer. Methods: This was a phase II, Simon 2-stage clinical trial in patients with biochemically recurrent prostate cancer with a primary endpoint of prostate-specific antigen (PSA) response. Patients were asymptomatic, with a rising PSA of at least $0.2 \mathrm{ng} / \mathrm{mL}$, and were treated with twice daily intake of Acai Juice Product until PSA progression, with a primary endpoint of PSA response. Results: Twenty-one patients were enrolled in the first stage of the trial. One of those patients had a PSA response within the study time period. The PSA doubling time was lengthened in $71 \%$ of patients $(95 \%$ confidence interval $=48 \%$ to $89 \%$ ) on the trial, and in a small number of responders, this was sustained over an extended time. Conclusions: This study did not meet its primary endpoint of $50 \%$ PSA response. Nevertheless, the overall tolerability and effects on PSA stabilization warrant further exploration in a biochemically recurrent population.
\end{abstract}

\section{Keywords}

prostate cancer, acai, biochemical recurrence, nutraceutical, clinical trial

Submitted March 22, 2018; revised August 17, 2018; accepted August 21, 2018

\section{Introduction}

Prostate cancer accounts for almost 165000 new cases and 27000 deaths each year in the United States. ${ }^{1}$ Primary management for patients with localized cancer generally includes active surveillance, radical prostatectomy, or radiation. As many as one third of men receiving definitive therapy, with radical prostatectomy or radiation for their prostate cancer, will relapse. ${ }^{2}$ The optimal timing of initiating androgen deprivation therapy (ADT) in patients with biochemically recurrent prostate cancer is still controversial because the use of early ADT in biochemically recurrent, nonmetastatic disease does not decrease prostate-specific mortality. ${ }^{3}$ Historical data suggest that in some men it may take up to a decade from the time of biochemical recurrence to the development of metastatic disease. ${ }^{4}$ Providers use baseline prostate-specific antigen (PSA), PSA velocity and doubling time, and Gleason score at diagnosis to help guide their estimate of a patient's disease biology within these contexts and the subsequent risk of metastatic disease in the short term. Patients may be hesitant to begin ADT due to numerous short- and longterm effects. However, continued observation may cause significant anxiety for many patients. Therefore, treatment with a nutraceutical may help provide a more tolerable approach to treat prostate cancer in situations where continued observation is an acceptable standard.

The field of nutraceuticals incorporates the use of a food or its components to provide health benefits. Plant derivatives, such as soy, ${ }^{5}$ grape seed extract, ${ }^{6}$ pomegranate, ${ }^{7-10}$ milk thistle, ${ }^{11-14}$ grape skin, ${ }^{15,16}$ and lycopene, ${ }^{17}$ have been studied as therapies for prostate cancer based on their

\footnotetext{
'University of Colorado, Aurora, CO, USA

Corresponding Author:

Elizabeth R. Kessler, Division of Medical Oncology, University of Colorado School of Medicine, MS 8I I7, I280I E 17th Avenue, Aurora, CO 80045, USA.

Email: Elizabeth.kessler@ucdenver.edu
} 
purported anti-inflammatory and antioxidant properties and low toxicities. These agents have most often been studied in patients with slowly rising PSA or biochemically recurrent disease, to try to slow the rate of PSA rise. The tolerability and suggestion of activity in prostate cancer provide an attractive option for prostate cancer patients. The acai berry, derived from a Brazilian palm tree, is rich in antioxidants such as anthocyanins and increases plasma antioxidant capacity up to 2.3 times when consumed as a juice. ${ }^{18}$ Preclinical work has demonstrated the free radical scavenging activity of this compound and its antiproliferative, proapoptotic effects on cancer cell lines. ${ }^{19}$ The bioflavonoids found in acai have been shown to induce apoptosis in preclinical studies using leukemia and esophageal cancer cell lines. ${ }^{20,21}$ While the mechanism of action is yet to be determined, there is interesting preclinical evidence both in vitro and in vivo to support clinical exploration. Additionally, anecdotal experience of 2 patients within our clinic with notable declines in PSA values while consuming acai juice prompted the evaluation of its efficacy in a clinical trial.

\section{Patients and Methods}

This was a phase II single-arm study with a Simon optimal design exploring the efficacy of Acai Juice Product (Eurobotanicals Inc, Fort Worth, TX) in patients with biochemically recurrent prostate cancer, or asymptomatic prostate cancer patients with a rising PSA (NCT01521949). Patients must have met the following basic eligibility criteria. Eligible patients had histologically proven adenocarcinoma of the prostate; evidence of rising PSA with a baseline PSA $\geqslant 0.2 \mathrm{ng} / \mathrm{mL}$; at least 3 PSA levels on record within a 6-month time period prior to enrollment in order to calculate baseline doubling time; Eastern Cooperative Oncology Group performance status 0 to 2 ; and adequate hematologic, renal, and hepatic function. Patients who were candidates for salvage local therapy must have had this option pursued, or at least discussed with them and declined, in order to be eligible. Patients could not be on any systemic antiandrogen or anticancer therapies and must have had a circulating testosterone level $>50 \mathrm{ng} / \mathrm{dL}$. Patients were excluded, or considered ineligible, if they had symptomatic disease, radiographically apparent metastatic disease, castration-resistant disease, or other uncontrolled intercurrent illness. Concurrent herbal therapies were prohibited, and any pretrial vitamin or mineral products were maintained at a stable dose throughout the trial.

The Acai Juice Product is a mixture of tea extracts and fruit juices, with $80 \%$ of the juice coming from the acai berry. The product is manufactured at a single facility with each batch tested for consistent oxygen radical absorbance capacity (ORAC). The juice product contains the following ingredients: Euterpe oleracea (acai) extract, white grape juice concentrate, cranberry apple syrup, concord grape juice concentrate, pear juice concentrate, passion fruit juice concentrate, cranberry juice concentrate, dark sweet cherry juice concentrate, Euterpe oleracea (acai) extract (from freeze dried powder), Coffea arabica (coffee) berry extract, and concentrated fruit extracts/powders of the following: wild blueberries, grapes, grape seeds, raspberries, raspberry seeds, cranberries, prunes, cherries, strawberries, and wild bilberries (VitaBerry Plus high-ORAC fruit blend); Camellia sinensis (green tea) leaf extract, Camellia sinensis (white tea) leaf extract, Aloe barbadensis leaf extract; Punica granatum (pomegranate) extract, ascorbic acid, and potassium. The recommended dose of the product is 2 ounces (oz) daily, and thus, this was the chosen dose for the study. In addition, this was the dose used by the 2 patients with anecdotal responses.

\section{Study Design}

Patients underwent initial screening and were then followed for up to 36 weeks at weeks $2,6,12,18,24$, and 36 . Treatment consisted of consumption of $2 \mathrm{oz}$ of Acai Juice Product by mouth twice daily for 36 weeks. Serum measurements of PSA, testosterone, chemistry, and complete blood count were taken at study initiation and completion. PSA values up to 300 days prior to treatment were collected from medical records. Baseline PSA is defined as the PSA value at start of Acai Juice Product treatment. Additionally, serum erythrocyte sedimentation rate (ESR) and C-reactive protein (CRP) were measured at baseline, 2 weeks, and 6 weeks. Adverse events were graded according to the National Cancer Institute Common Terminology Criteria for Adverse Events (CTCAE v 4.0). After receipt of an investigational new drug exemption by the Food and Drug Administration, the protocol was approved by the local institutional review board and conducted in accordance with the Declaration of Helsinki.

The primary endpoint was the proportion of patients with PSA response, defined as PSA decrease of $\geqslant 50 \%$ from baseline, confirmed by a PSA value obtained $\geqslant 6$ weeks later. Secondary endpoints included PSA doubling time, PSA velocity, and duration of PSA response. Progressive disease was defined as a $25 \%$ or greater increase from PSA nadir in addition to an absolute increase of $\geqslant 2 \mathrm{ng} / \mathrm{mL}$, which was confirmed by a second value obtained more than 6 weeks later. There were no required imaging studies. The study was powered to detect a PSA response in $\geqslant 20 \%$ patients (against $\leqslant 5 \%$ ) at $90 \%$ power.

\section{Laboratory Correlates}

Samples for correlative laboratory analysis were collected at baseline, 2 weeks, and 6 weeks for analysis of the mechanism of Acai Juice Product. After collection, samples were centrifuged for 15 minutes at $1000 \times g$ to collect plasma. 
De-identified plasma samples were then stored as $1 \mathrm{~mL}$ aliquots at $-80^{\circ} \mathrm{C}$. Assays were run using the $\mathrm{M} 30$ Apoptosense ELISA to measure caspase-cleaved keratin18 (ccK18) in plasma, and the Millipore Human Cytokine array was used to measure cytokine levels in plasma. Caspases are activated in apoptotic cells and cleave intracellular protein substrates. K18 is one such substrate, expressed by many epithelial cells (eg, hepatocytes, intestinal cells, breast cells, and prostate cells). Cleaved K18 is released into the circulation after cell death. M30 Apoptosense ELISA is an enzymelinked immunosorbent assay developed for the detection of soluble caspase-cleaved keratin 18 (ccK18, K18-Asp396, formerly cytokeratin 18, ccCK18, or CK18-Asp396). The M30 Apoptosense ELISA is a noninvasive test for the detection of apoptosis of epithelially derived cells.

\section{Statistical Analysis}

The Simon optimal design was used to test the null hypothesis that $P<.05$ versus the alternative that $P=.20$. A maximum of 41 subjects were planned for enrollment. Twenty-one subjects were enrolled during stage I; if $>1$ patient demonstrated a PSA response then the study would proceed to the second stage, with enrollment of an additional 20 patients. An exact binomial test was used to assess whether the proportion of patients with PSA response was different from 5\%. For each individual patient, linear regression was used to estimate PSA velocity, which mathematically is the slope of PSA growth line; velocity was then used to calculate PSA doubling time (DT): DT = PSA baseline/velocity. We calculated DT both prior to and after acai juice treatment. Based on the results of linear regression, we also calculated predicted PSA values for each patient, pretreatment and posttreatment (Figure 1). Percentage of maximum PSA change, defined as (lowest posttreatment PSA - baseline PSA)/baseline PSA $\times 100 \%$, was also calculated for each patient (Figure 2).

\section{Results}

Twenty-one patients were enrolled between November 2011 and November 2012 at the University of Colorado Urologic Oncology Clinic (Table 1). The majority (71\%) of patients had Gleason 7 cancers, 14\% had Gleason 5 to 6, and 19\% with Gleason 8 to 10 . The median PSA at study entry was $2.74 \mathrm{ng} / \mathrm{mL}$, with a range of 0.33 to $36.9 \mathrm{ng} / \mathrm{mL}$. Five patients had received prior ADT with concurrent radiation, with a window of at least 1 year since completion of ADT.

The primary endpoint, PSA response $>50 \%$, was seen in $1 / 21$ patients within the defined treatment period of 30 weeks. The study team originally planned for patients to remain on juice product as long as feasible; however, the supply of study juice product was limited as the study progressed, and thus, the treatment period was defined as 30 weeks. This is an adequate time to see a response in PSA in

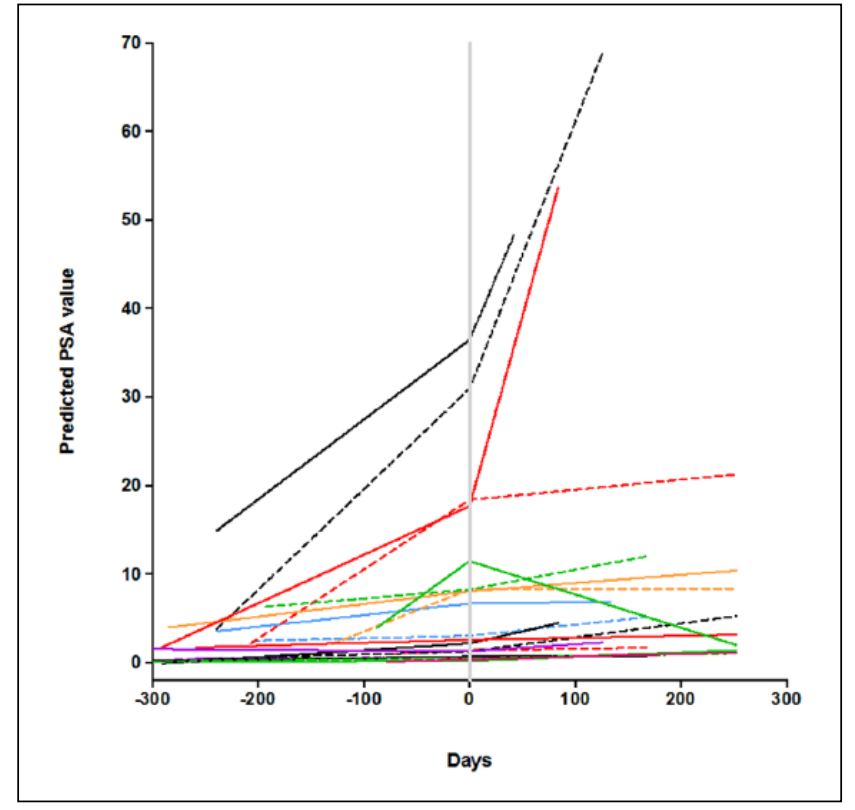

Figure I. PSA doubling time. Time 0 is start of Acai Juice Product. Each line represents a different patient. Patients had at least 2 pretreatment PSA tests to inform the slope and then a 6-month follow-up PSA as well.

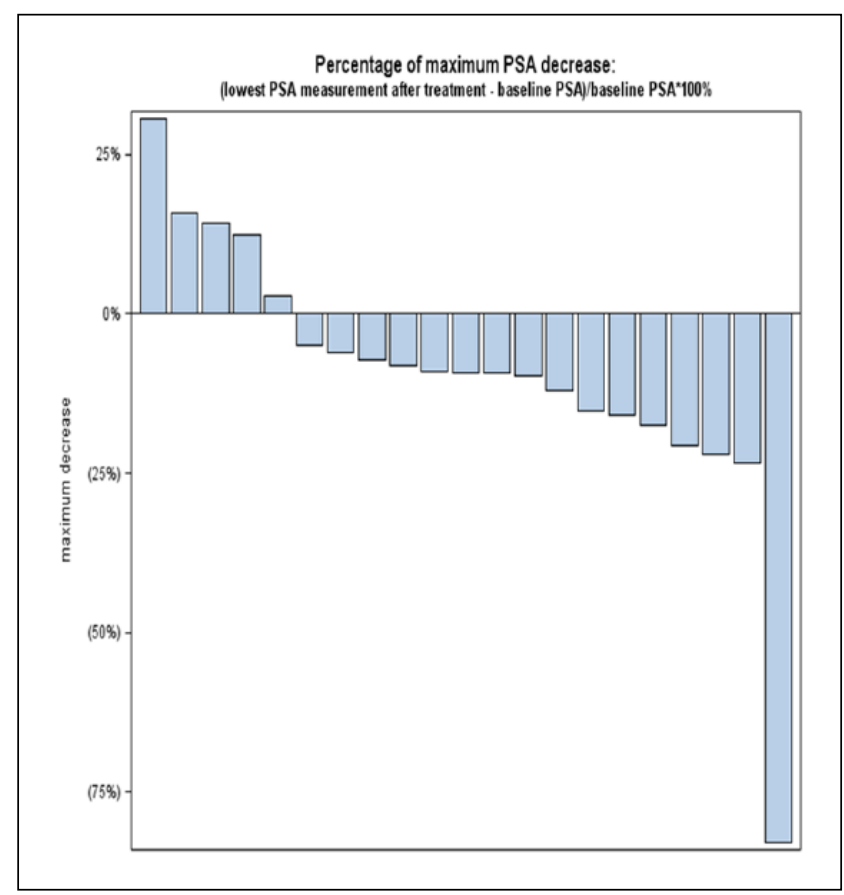

Figure 2. Maximal decrease of PSA while on study. Each bar represents patients with the value correlating to the best PSA change from baseline while on study.

patients with prostate cancer. Beyond the reported study responder, a second patient chose to remain on the juice product after the study had completed. He also experienced 
Table I. Baseline Patient Characteristics.

\begin{tabular}{lc}
\hline Number of patients & 2 I \\
Median age (range) & $69(54-80)$ \\
Baseline ECOG & 6 \\
0 & 15 \\
I & \\
Gleason score (\%) & $3(14 \%)$ \\
6 & $14(67 \%)$ \\
7 & $4(19 \%)$ \\
$8-10$ & $2.74(0.33-43.4)$ \\
Baseline PSA (range) & \\
Previous therapy-may have had multiple & 12 \\
Radical prostatectomy & 11 \\
External beam radiation & 5 \\
$\quad$ With concurrent ADT & 3 \\
Brachytherapy & 2 \\
$5-\alpha$ reductase inhibitor & 4 \\
ADT & 1 \\
Antiandrogen & $6(3-20)$ \\
Years since diagnosis median (range)
\end{tabular}

Abbreviations: ECOG, Eastern Cooperative Oncology Group; PSA, prostate-specific antigen; ADT, androgen deprivation therapy.

a decline of $>50 \%$ from PSA baseline/nadir; however, this occurred at week 36 and could not be considered for endpoint analysis. The study was terminated after stage I for futility. All 21 patients enrolled in study received at least one dose of the Acai Juice Product, and were therefore included in the statistical analysis.

The PSA doubling time was lengthened in $71 \%$ patients $(95 \%$ confidence interval $=48 \%$ to $89 \%)$ on the trial. Figure 1 represents the calculated PSA based on doubling time: each patient is represented as a single line, and there is evidence of a slowing of the PSA doubling time in multiple subjects as well as a decline in some patients. While only 1 of 21 patients had a PSA response as defined by study endpoints, 15 patients had a PSA decline from baseline. The best PSA response on study is represented in Figure 2. No study related adverse events were reported.

Additional laboratory correlative analysis was performed on patient plasma samples in order to investigate the potential mechanism of action of the Acai Juice Product. Patients served as their own baseline. Seven of the 21 patients had CRP and ESR analysis at all time points. Changing PSA values did not correlate with changing CRP or ESR. A marker of apoptosis is ccK18. An increase from baseline in ccK18 was seen at 2 weeks in a majority of patients (13/19). The one clinical responder on trial had a significantly higher level of ccK18 throughout the trial with a peak at week 6 . However, baseline ccK18 values did not correlate with clinical outcome. Millipore Human Cytokine array did not reveal any significant signals in the samples (data not shown).

\section{Discussion}

This study of 21 men with biochemically recurrent prostate cancer treated with Acai Juice Product did not result in marked PSA responses, but did show benefit through secondary endpoints of PSA doubling time and velocity. While this Simon 2-stage trial did not progress beyond the first stage, a PSA decline of $\geqslant 50 \%$ was a fairly ambitious endpoint for this patient population. The endpoint was developed based on observation of marked PSA responses in 2 patients taking the product independently prior to the development of this protocol.

Most patients had a prolongation of PSA doubling time while on study, but it is difficult to discern if this was a reflection of disease natural history, PSA variability, or a treatment effect. The study population included patients with a variety of Gleason scores, pretreatments, and baseline PSA suggesting a variety of disease biology. There was no clear correlation between these characteristics and response on trial. In the most advanced stages of prostate cancer, changes in PSA are less indicative of treatment response in comparison with radiographic changes. ${ }^{22}$ At the same time, PSA change and doubling time are often meaningful endpoints in trials of earlier stage or biochemically recurrent disease. The Prostate-Specific Antigen Working Group's guidelines on doubling time support this as a tool to predict clinical progression for patients with biochemically recurrent disease based on available evidence. ${ }^{23}$ Retrospective analysis suggests that the doubling time predicts likelihood of the disease becoming symptomatic or life-threatening. ${ }^{24}$ The clinical importance of these measures and proper methods of calculation are often debated. ${ }^{25,26}$ Additionally, while there is suggestion of clinical utility in a change in doubling time, the single-arm design of this study limits the interpretation of change in doubling time as a signal of an active therapy. ${ }^{25}$ In a population of patients with biochemical recurrence only, a minimally toxic therapy that has potential to slow the growth of disease seems important, and this may be reflected in doubling time.

In addition to this work, other placebo-controlled nutraceutical studies using pomegranate juice or muscadine grape extract report a lengthening of doubling time in both placebo and treatment arms. ${ }^{7,8,27}$ More recent studies have focused on predicting those patients who may experience an exceptional PSA response. These studies have identified potentially predictive genotypes of response. Similarly, this study of acai juice extract also had a limited number of exceptional responders.

Additional correlative analyses were performed to discern the potential mechanisms of action as less preclinical investigation is available for acai in comparison with other nutraceuticals. In investigating an inflammatory response, well-known serum assays that may serve as 
surrogate markers of inflammation (ie, CRP, ESR) were explored in a limited number of participants, without a relationship between response and these values. Similarly, the inflammatory activity was evaluated via cytokine analysis with no correlation noted. In order to better identify mechanisms of action of the acai plant, the team analyzed levels of soluble caspase-cleaved keratin 18 (cck18). These data suggest that the effect of the product may be through apoptosis. The cck18 assay is not validated for clinical use or evaluation, but has been evaluated in other tumor types with suggestion that an increase in circulating ccK18 may signify tumor response in those tumors as well. ${ }^{28,29}$

The role of lifestyle modification in preventing and controlling cancer is an active topic of investigation. In hormonally mediated cancers, such as breast cancer and prostate cancer, dietary habits hold importance in risk of diagnosis as well as recurrence. ${ }^{30-32} \mathrm{~A}$ large cooperative group trial by the Cancer and Leukemia Group B is evaluating men's dietary habits during active surveillance in order to gathermore information on this interplay (NCT01238172). Currently, some of our most effective treatments rely on the same tenet as ADT - to minimize the androgen axis - and yet we are also aware of the long-term side effects associated with this approach, and the need to evaluate mechanisms of treatment with perhaps better toxicity profiles. Nutraceuticals may be an interesting approach to patients with biochemical recurrence and no detectable metastatic disease, in whom disease symptoms or radiographic disease may not develop for months to years. Of note, our trial accrued quickly, with a waiting list of patients at the time of study closing. This suggests that patients may prefer this class of therapy in order to postpone, or avoid altogether, the toxicities of ADT. There is a dearth of studies in this disease stage, and yet a strong need and interest as identified by patient involvement in this study.

While this study did not reach its primary end point of PSA response $>50 \%$, it did show modest decrease in PSA and slowing of doubling time in many patients. Studies in other nutraceuticals have shown a lengthening of the PSA doubling time in both the treatment and placebo groups. ${ }^{9}$ The robust response in a few patients in this study suggests that there may be an additional genomic predictive marker, such as the genetic polymorphism associated with response to both pomegranate juice and muscadine grape skin extract. ${ }^{8,9,15,16}$

\section{Conclusion}

This study did not meet its primary endpoint. Nevertheless, the overall tolerability and effects on PSA stabilization warrant further exploration especially in the proper selection of patients. This small single-arm study requires larger study but does suggest a role for acai in stabilizing disease.

\section{Acknowledgments}

Ken McKinney; Eurobotanicals, Inc; NCI \#2P30CA046934-24; Lam-K12CA086913 (Paul Calabresi Clinical Oncology Scholars Award).

\section{Declaration of Conflicting Interests}

The author(s) declared no potential conflicts of interest with respect to the research, authorship, and/or publication of this article.

\section{Funding}

The author(s) disclosed receipt of the following financial support for the research, authorship, and/or publication of this article: They received financial support from the following non-profit foundations for the research of this article: William R. Meyn Foundation; William Mann Foundation.

\section{References}

1. Siegel RL, Miller KD, Jemal A. Cancer statistics, 2016. $C A$ Cancer J Clin. 2016;66:7-30.

2. Roehl KA, Han M, Ramos CG, Antenor JA, Catalona WJ. Cancer progression and survival rates following anatomical radical retropubic prostatectomy in 3478 consecutive patients: long-term results. J Urol. 2004;172:910-914.

3. Studer UE, Whelan $\mathrm{P}$, Albrecht W, et al. Immediate or deferred androgen deprivation for patients with prostate cancer not suitable for local treatment with curative intent: European Organisation for Research and Treatment of Cancer (EORTC) Trial 30891. J Clin Oncol. 2006;24:1868-1876.

4. Pound CR, Partin AW, Eisenberger MA, Chan DW, Pearson JD, Walsh PC. Natural history of progression after PSA elevation following radical prostatectomy. JAMA. 1999;281: 1591-1597.

5. Hussain M, Banerjee M, Sarkar FH, et al. Soy isoflavones in the treatment of prostate cancer. Nutr Cancer. 2003;47: 111-117.

6. Raina K, Singh RP, Agarwal R, Agarwal C. Oral grape seed extract inhibits prostate tumor growth and progression in TRAMP mice. Cancer Res. 2007;67:5976-5982.

7. Pantuck AJ, Zomorodian N, Belldegrun AS. Phase-II study of pomegranate juice for men with prostate cancer and increasing PSA. Curr Urol Rep. 2006;7:7.

8. Paller CJ, Ye X, Wozniak PJ, et al. A randomized phase II study of pomegranate extract for men with rising PSA following initial therapy for localized prostate cancer. Prostate Cancer Prostatic Dis. 2013;16:50-55.

9. Pantuck AJ, Pettaway CA, Dreicer R, et al. A randomized, double-blind, placebo-controlled study of the effects of pomegranate extract on rising PSA levels in men following primary therapy for prostate cancer. Prostate Cancer Prostatic Dis. 2015;18:242-248.

10. Thomas R, Williams M, Sharma H, Chaudry A, Bellamy P. A double-blind, placebo-controlled randomised trial evaluating the effect of a polyphenol-rich whole food supplement on PSA progression in men with prostate cancer- the UK NCRN Pomi-T study. Prostate Cancer Prostatic Dis. 2014;17:180-186. 
11. Flaig TW, Su LJ, Harrison G, Agarwal R, Glode LM. Silibinin synergizes with mitoxantrone to inhibit cell growth and induce apoptosis in human prostate cancer cells. Int $J$ Cancer. 2007; 120:2028-2033.

12. Flaig TW, Gustafson DL, Su LJ, et al. A phase I and pharmacokinetic study of silybin-phytosome in prostate cancer patients. Invest New Drugs. 2007;25:139-146.

13. Flaig TW, Glode M, Gustafson D, et al. A study of high-dose oral silybin-phytosome followed by prostatectomy in patients with localized prostate cancer. Prostate. 2010;70:848-855.

14. Agarwal R, Goel SK, Behari JR. Detoxification and antioxidant effects of curcumin in rats experimentally exposed to mercury. J Appl Toxicol. 2010;30:457-468.

15. Paller CJ, Rudek MA, Zhou XC, et al. A phase I study of muscadine grape skin extract in men with biochemically recurrent prostate cancer: safety, tolerability, and dose determination. Prostate. 2015;75:1518-1525.

16. Paller CJ, Zhou XC, Heath EI, et al. Muscadine grape skin extract (MPX) in men with biochemically recurrent prostate cancer: a randomized, multicenter, placebo-controlled clinical trial. Clin Cancer Res. 2018;24:306-315.

17. Paur I, Lilleby W, Bøhn SK, et al. Tomato-based randomized controlled trial in prostate cancer patients: effect on PSA. Clin Nutr. 2017;36:672-679.

18. Mertens-Talcott SU, Rios J, Jilma-Stohlawetz P, et al. Pharmacokinetics of anthocyanins and antioxidant effects after the consumption of anthocyanin-rich acai juice and pulp (Euterpe oleracea Mart.) in human healthy volunteers. J Agric Food Chem. 2008;56:7796-7802.

19. Schauss AG, Wu X, Prior RL, et al. Antioxidant capacity and other bioactivities of the freeze-dried Amazonian palm berry, Euterpe oleraceae mart. (acai). J Agric Food Chem. 2006;54:8604-8610.

20. Del Pozo-Insfran D, Percival SS, Talcott ST. Acai (Euterpe oleracea Mart.) polyphenolics in their glycoside and aglycone forms induce apoptosis of HL-60 leukemia cells. J Agric Food Chem. 2006;54:1222-1229.

21. Stoner GD, Wang LS, Seguin C, et al. Multiple berry types prevent N-nitrosomethylbenzylamine-induced esophageal cancer in rats. Pharm Res. 2010;27:1138-1145.

22. Scher HI, Halabi S, Tannock I, et al. Design and end points of clinical trials for patients with progressive prostate cancer and castrate levels of testosterone: recommendations of the Prostate Cancer Clinical Trials Working Group. J Clin Oncol. 2008;26:1148-1159.

23. Arlen PM, Bianco F, Dahut WL, et al. Prostate Specific Antigen Working Group guidelines on prostate specific antigen doubling time. J Urol. 2008;179:2181-2185.

24. D'Amico AV, Moul J, Carroll PR, Sun L, Lubeck D, Chen $\mathrm{MH}$. Prostate specific antigen doubling time as a surrogate end point for prostate cancer specific mortality following radical prostatectomy or radiation therapy. J Urol. 2004;172(5 pt 2):S42-S46.

25. Paller CJ, Olatoye D, Xie S, et al. The effect of the frequency and duration of PSA measurement on PSA doubling time calculations in men with biochemically recurrent prostate cancer. Prostate Cancer Prostatic Dis. 2014;17: 28-33.

26. Svatek RS, Shulman M, Choudhary PK, Benaim E. Critical analysis of prostate-specific antigen doubling time calculation methodology. Cancer. 2006;106:1047-1053.

27. Pantuck AJ, Leppert JT, Zomorodian N, et al. Phase II study of pomegranate juice for men with rising prostate-specific antigen following surgery or radiation for prostate cancer. Clin Cancer Res. 2006;12:4018-4026.

28. Ulukaya E, Karaagac E, Ari F, et al. Chemotherapy increases caspase-cleaved cytokeratin 18 in the serum of breast cancer patients. Radiol Oncol. 2011;45:116-122.

29. Chu TQ, Ding H, Garfield DH, et al. Can determination of circulating endothelial cells and serum caspase-cleaved CK18 predict for response and survival in patients with advanced non-small-cell lung cancer receiving endostatin and paclitaxel-carboplatin chemotherapy? A retrospective study. $J$ Thorac Oncol. 2012;7:1781-1789.

30. Ornish D, Weidner G, Fair WR, et al. Intensive lifestyle changes may affect the progression of prostate cancer. J Urol. 2005; 174:1065-1069.

31. Gong Z, Neuhouser ML, Goodman PJ, et al. Obesity, diabetes, and risk of prostate cancer: results from the prostate cancer prevention trial. Cancer Epidemiol Biomarkers Prev. 2006;15:1977-1983.

32. Cohen JH, Kristal AR, Stanford JL. Fruit and vegetable intakes and prostate cancer risk. J Natl Cancer Inst. 2000;92: 61-68. 